psychopathology. We addressed limitations of the meta-analyses in our original paper. We suggest that careful and comprehensive examination of the diverse phenotypes associated with neuropsychiatric illness may be a more fruitful approach.

Second, Dr Crow cites his own review of the linkage literature to suggest that most of the candidate genes reported by our group, and many others, are not supported by linkage studies and thus should be discounted. This reasoning is based on a flawed understanding of the role of linkage in complex disorders and is inconsistent with a large body of recent empirical evidence in complex genetics. In other complex disorders, a majority of susceptibility loci that have been unambiguously replicated in association studies fall outside of previously identified areas of even suggestive linkage (e.g. Barrett et $a l^{1}$ ). Therefore, an argument utilising non-significant linkage data to invalidate a subsequent candidate gene association is erroneous.

Third, Dr Crow notes the productivity of our lab over the past several years as a source of concern for him. In so doing he mischaracterises our papers. First, he is simply incorrect in stating that only one paper reports strictly negative results (see Fubke et $a l^{2}$ and Hodgkinson $e t a l^{3}$ ). Moreover, many of our papers report complex relationships that are not so simplistically reduced to 'positive' v. 'negative'. More importantly, Dr Crow fails to mention that most of our papers are not simply analyses of association to schizophrenia diagnosis, but instead examine alternative phenotypes. For example, our study of DRD2 assessed the relationship between a functional promoter region polymorphism and clinical response to olanzapine and risperidone in the context of a randomised controlled clinical trial in first-episode schizophrenia. ${ }^{4}$ Therefore, it is not surprising that our DRD2 results were not 'replicated' in either linkage studies or the association study of Sanders et $a l,{ }^{5}$ as these papers were restricted to mere association to diagnosis.

Although Dr Crow is entitled to his opinions, the field of psychiatric genetics may be better served by more constructive discussion leading towards a better understanding of the complexities of these devastating disorders.

1 Barrett JC, Hansoul S, Nicolae DL, Cho JH, Duerr RH, Rioux JD, et al. Genomewide association defines more than 30 distinct susceptibility loci for Crohn's disease. Nat Genet 2008; 40: 955-62.

2 Funke BH, Lencz T, Finn CT, DeRosse P, Poznik GD, Plocik AM, et al. Analysis of TBX1 variation in patients with psychotic and affective disorders. Mol Med 2007; 13: 407-14.

3 Hodgkinson CA, Goldman D, Ducci F, DeRosse P, Caycedo DA, Newman ER, et al. The FEZ1 gene shows no association to schizophrenia in Caucasian or African American populations. Neuropsychopharmacology 2007; 32: 190-6.

4 Lencz T, Robinson DG, Xu K, Ekholm J, Sevy S, Gunduz-Bruce H, et al. DRD2 promoter region variation as a predictor of sustained response to antipsychotic medication in first-episode schizophrenia patients. Am J Psychiatry 2006; 163: 529-31.

5 Sanders AR, Duan J, Levinson DF, Shi J, He D, Hou C, et al. No significant association of 14 candidate genes with schizophrenia in a large European ancestry sample: implications for psychiatric genetics. Am J Psychiatry 2008; 165: $497-506$

Todd Lencz, The Zucker Hillside Hospital, Psychiatry Research, 75-59 263rd Street, Glen Oaks, New York 11004, USA. Email: lencz@lij.edu; Robert H. Lipsky, Laboratory of Neurogenetics, National Institute on Alcohol Abuse and Alcoholism, Bethesda; Katherine E. Burdick, Center for Translational Psychiatry, Feinstein Institute for Medical Research and Division of Psychiatry Research, The Zucker Hillside Hospital, New York; David Goldman, Colin Hodgkinson, Section of Human Neurogenetics, National Institute on Alcohol Abuse and Alcoholism, Rockville; John M. Kane, Center for Translational Psychiatry, Feinstein Institute for Medical Research and Department of Psychiatry, The Zucker Hillside Hospital, New York; Anil K. Malhotra, Center for Translational Psychiatry, Feinstein Institute for Medical Research and Division of Psychiatry Research, The Zucker Hillside Hospital, New York, USA

doi: 10.1192/bjp.195.2.179a

\section{Outcome of group psychoeducation for stabilised bipolar disorders}

The article by Colom et $a l^{1}$ further enhanced our understanding about the role of psychoeducation in the management of bipolar disorders. The study draws its strengths from the fact that it included an active control group and individuals with bipolar disorder and Axis II comorbidity, follow-up rates were excellent and the authors assessed the outcome in the form of the number and type of recurrences, time to recurrence, time spent ill and number of hospitalisations at 5 years. However, some of the issues require further clarification.

When one looks at the article reporting 2-year follow-up of the same cohort, ${ }^{2}$ the authors report that individuals with Axis I comorbidity were excluded, but at 5-year follow-up the authors report that only those with severe Axis I diagnosis were excluded. Further, the authors do not define 'severe'. Individuals with bipolar disorder can have a high rate of comorbidity, hence clarification of this fact is very important from the perspective of generalisability of the study findings. In addition, Colom et al do not provide details of status and/or type of Axis I/II comorbidities and whether the drop-out rate and the number of completers made any difference with regard to clinical and demographic features.

Another important aspect is the way the authors defined recurrence based on rating scale scores. This type of definition in the true sense does not include the subsyndromal symptoms and can influence almost all the outcome measures such as time spent ill, time to recurrence and the number of recurrences, especially when the cohort is being followed up at a frequency of every 2 weeks. Similarly, although the study included the number and duration of hospitalisations as an outcome measure, the authors have not discussed the criteria for hospitalisation.

Another important aspect which needs clarification is the analysis of data. In many places Colom et al have used parametric tests to compare the numerical variables, although the standard deviation is more than the mean. Similarly, mean values are given for the number of recurrences without standard deviations, and comparison statistics are given as F-values. In Table 2, ${ }^{1}$ again the authors compare the mean values using Fisher $F$ statistics and demonstrate that there was a significant difference in the number of days spent in each episode for all types of episodes. However, when one looks at the data, it is difficult to understand this contention. In the same table when one adds the mean number of days spent in each episode for the control group, the data regarding each episode and the total duration do tally, but the same is not the case for the psychoeducation group.

1 Colom F, Vieta E, Sánchez-Moreno J, Palomino-Otiniano R, Reinares M, Goikolea JM, et al. Group psychoeducation for stabilised bipolar disorders: 5year outcome of a randomised clinical trial. Br J Psychiatry 2009; 194: 260-5.

2 Colom F, Vieta E, Martinez-Aran A, Reinares M, Goikolea JM, Benabarre A, et al. A randomized trial on the efficacy of group psychoeducation in the prophylaxis of recurrences in bipolar patients whose disease is in remission. Arch Gen Psychiatry 2003; 60: 402-7.

Navendu Gaur, Department of Psychiatry, Postgraduate Institute of Medical Education and Research, Chandigarh, India. Email: drsandeepg2002@yahoo.com Sandeep Grover, Department of Psychiatry, Postgraduate Institute of Medical Education and Research, Chandigarh, India

doi: 10.1192/bjp.195.2.180

Authors' reply: We would like to provide some clarifications in response to Gaur \& Grover's queries.

First, only those patients with 'severe' Axis I comorbidity diagnoses were excluded. This means that patients were excluded if 
they presented with a coexisting Axis I condition that might have a major impact on their ability to effectively participate in the groups, such as severe social phobia or obsessive-compulsive disorder.

Second, regarding details of status and/or type of Axis I/II comorbidities, we would like to point out that this was already covered for the 2-year follow-up in a previous paper. ${ }^{1}$

Third, we defined recurrence both based on severity ratings and DSM-IV criteria; these are narrow criteria which are much more reliable than just asking for diagnostic criteria alone or rating scale scores. We disregarded the possibility of using a lifechart method to catch subsyndromal fluctuations because this method has not shown good reliability and would likely capture a lot of noise.

Fourth, criteria for hospitalisation were those used at the Barcelona Bipolar Disorders Program: any patient presenting an episode that, owing to its severity, cannot be managed in an out-patient setting and/or any patient presenting suicide risk or representing a risk for third persons.

Fifth, as clearly explained in our manuscript, the primary outcome of the trial was time to recurrence. Secondary outcomes included time spent ill and number of recurrences. Our original submission included a full data report on those secondary variables, which had to be condensed owing to space constraints. The analysis of the number of recurrences was, as explained in the Method, performed by means of ANCOVA and therefore the mean values for each group are just orientive.

Finally, we acknowledge a typing error in Table 2 referring to the number of days spent in depression. The right values should be: control group, mean $=398.55$ days $($ s.d. $=364.16)$; psychoeducation group, mean $=93.28$ days $($ s.d. $=165.46)$. The standard deviation for the control group was mistakenly repeated replacing the mean number of days spent in depression for the psychoeducation group. After correcting this error, data regarding mean number of days spent in each episode tally with the total duration for both groups. As this was only a typing error, it does not change any statistics. We have been informed of this mistake by other readers and have already proceeded to issue the corresponding erratum.

1 Colom F, Vieta E, Sánchez-Moreno J, Martínez-Arán A, Torrent C, Reinares M, et al. Psychoeducation in bipolar patients with comorbid personality disorders. Bipolar Disord 2004; 6: 294-8.

Francesc Colom, Bipolar Disorders Program, Hospital Clinic of Barcelona, Villarroel 170, 08036 Barcelona, Spain. Email: fcolom@clinic.ub.es; Eduard Vieta, Bipolar Disorders Program, Institute of Neurosciences, Hospital Clinic, IDIBAPS, CIBER-SAM, University of Barcelona, Spain

doi: 10.1192/bjp.195.2.180a

\section{Abortion and mental health: established facts reconsidered}

Tyrer's 'From the Editor's desk' lyrically asserted that in relation to the paper by Fergusson et $a l^{1}$ and other studies, 'In the parched desert of ignorance and prejudice every established fact becomes an oasis. By "established fact" I mean one that defines the field, the one that all the related and restlessly inchoate facts gather round and say "I belong here", and then fall into line behind it."

Fergusson $e a^{1}$ conclude that there is evidence that abortion may be associated with a small increase in risk of mental disorders and in comparison, other pregnancy outcomes were not associated with increased risk. Although we acknowledge that aspects of their analytic design are strong and carefully implemented, we believe that the analyses have not maximised the potential of the dataset and that therefore, your editor's rhetorical confidence is not yet justified. We advance the following reasons.
First, Fergusson et al dichotomised each pregnancy exposure. Of 534 women in the Christchurch cohort, 284 had had pregnancies. Women making decisions about terminating pregnancies may have prior pregnancy events and potentially cumulative losses will have different mental health impacts compared with termination as the outcome of a first pregnancy. Pregnancy variables are not independent and mutual adjustment in models for other outcomes will not account for the interactions between pregnancy outcomes. A more useful analysis would have been with a composite variable with never having had a pregnancy event as the reference category.

Second, the combining of therapeutic abortion for fetal malformation with abortion by choice is inappropriate. Most abortions are first trimester. There is an argument for separating termination of pregnancy by gestational age, so that the mental health impact of those in the second or third trimester are visible and separate. It is possible that terminating a wanted pregnancy because of fetal abnormality would be more distressing than an early unwanted pregnancy.

Third, many authors (including Fergusson et al) have found strong relationships between intimate partner violence and poor mental health, and between intimate partner violence and increased association with reporting terminations. ${ }^{3-6}$ Despite the potential to include the much more rigorous measure from their previous study of partner violence among this cohort, the authors have excluded their strongest measures of partner violence in this analysis, leaving a major covariate poorly measured.

Fergusson et al conclude that the evidence for abortion impact is small but clear - even causal. Yet there is no evidence that the risks associated with other pregnancy outcomes, particularly loss, are different from those estimated for abortion (see Charles et $\mathrm{l}^{7}$ ), nor that mental health disorders are incident after an abortion. This could have been statistically tested using logistic regression among the range of statistical tests already carried out.

It is a pity that such a good cohort study has not been better analysed. With the above adjustments, the authors would be better placed to more clearly identify the vulnerable groups they are wisely seeking to identify.

1 Fergusson DM, Horwood LJ, Boden JM. Abortion and mental health disorders: evidence from a 30-year longitudinal study. Br J Psychiatry 2008; 193: 444-51.

2 Tyrer P. From the Editor's desk. Br J Psychiatry 2008; 193: 524.

3 Taft AJ, Watson LF. Depression and termination of pregnancy (induced abortion) in a national cohort of young Australian women: the confounding effect of women's experience of violence. BMC Public Health 2008; 8: 75.

4 Fergusson DM, Horwood L, Ridder EM. Partner violence and mental health outcomes in a New Zealand birth cohort. J Marriage Fam 2005; 67: 1103-19.

5 Hegarty KL, Gunn J, Chondros P, Small R. Association between depression and abuse by partners of women attending general practice: descriptive, cross-sectional survey. BMJ 2004; 328: 621-4.

6 Gazmararian JA, Adams MM, Saltzman LE, Johnson CH, Bruce FC, Marks JS, et al. The relationship between pregnancy intendedness and physical violence in mothers of newborns. The PRAMS Working Group. Obstet Gynecol 1995; 85: 1031-8.

7 Charles VE, Polis CB, Sridhara SK, Blum RW. Abortion and long term mental health outcomes: a systematic review of the evidence. Contraception 2008; 78: $436-50$.

Angela J. Taft, Mother and Child Health Research, La Trobe University, 324-328 Little Lonsdale Street, Melbourne, Austrlia. Email: a.taft@latrobe.edu.au; Lyndsey Watson, Mother and Child Health Research, La Trobe University, Australia

doi: 10.1192/bjp.195.2.181

Authors' reply: Taft \& Watson claim that we measured pregnancy history using dichotomous measures and that this fails to represent the complexities of pregnancy history. This claim misrepresents our analysis. 